\title{
Meningocele sacral anterior associada a fístula retotecal e meningite polimicrobiana: relato de caso
}

\author{
Rodrigo de Almeida Simon Sola', Bruno Leonardo Zappa Ferreira Gonçalves ${ }^{1}$, \\ André de Almeida e Silva', Juliano Nery Navarro', \\ Nilton Rocha da Silva Junior ${ }^{1}$, Leandro Gomes Brito', Luis Otavio Carneiro \\ Pontelli', Luiz Gustavo de Abreu Mattos ${ }^{1}$, João Flávio Mattos Araujo ${ }^{2}$
}

Serviço de Neurocirurgia do Hospital Celso Pierro da Pontifícia Universidade Católica de Campinas (PUC-Campinas), Campinas, SP, Brasil.

\section{RESUMO}

Meningoceles sacrais anteriores são exemplos raros de disrafismo espinhal, originados da herniação do saco dural, através de um defeito ósseo na parede anterior sacrococcígea. Essa condição foi descrita pela primeira vez em 1837 por Bryant, e existem, aproximadamente, 250 casos relatados até os dias atuais. A tríade defeito ósseo, malformação anorretal e massa pré-sacral configura a síndrome de Currarino, e tal massa pode consistir de um tumor, de uma meningocele sacral anterior ou da associação de ambos. Tal síndrome apresenta incidência desconhecida, e acredita-se que seja uma desordem de transmissão autossômica dominante. O diagnóstico ainda é desafiador, apesar da evolução dos exames de imagem. Tomografia computadorizada (TC) e ressonância magnética (RNM) são primordiais, sendo a última o melhor exame para caracterização. A radiografia simples pode ajudar demonstrando o defeito ósseo sacral e o sacro em "cimitarra", sendo indicada para triagem de transmissão familiar. A maioria dos pacientes apresenta constipação e sintomas vesicais compressivos. A meningite é uma complicação rara e séria da meningocele sacral anterior, e a associação entre fístula retotecal e pneumoencéfalo foi relatada em apenas dois artigos de língua inglesa. O tratamento deve ser cirúrgico, uma vez que não há possibilidade de fechamento espontâneo. Em casos não tratados ou com atraso diagnóstico, a mortalidade é superior a $30 \%$ quando associados à infecção. Descrevemos um caso de meningocele sacral anterior acometendo uma jovem de 17 anos, assintomática até então, que apresentou meningite polimicrobiana como quadro inicial devido a uma fístula retotecal.

\section{PALAVRAS-CHAVE}

Meningocele, fístula, meningite.

\section{ABSTRACT}

Anterior sacral meningocele associated with polymicrobial meningitis and rectothecal fistula: case report

Anterior sacral meningoceles are rare examples of spinal dysraphism, originated from the dural sac herniation through a bone defect in the anterior wall of the sacrococcygeal bone. This condition was first described in 1837, by Bryant, and there are, approximately, 250 cases reported until today. The triad bone defect, anorectal malformation and presacral mass configure the Currarino syndrome, and such mass may consist of a tumor, an anterior sacral meningocele or combination of both. The incidence is unknown and it is believed to be an autosomal dominant disorder. The diagnosis is still challenging, despite the evolution of imaging. CT and MRI are essential, although the MRI is the best test for characterization. Plain radiographs can help demonstrate the sacral bone defect and the "scimitar" sacrum, and they are suitable for familial transmission screening. Most patients have constipation and compressive bladder symptoms. Meningitis is a rare and severe complication of anterior sacral meningocele and association of rectothecal fistula and pneumocephalus was reported in only two articles in the English language. The surgical treatment must be performed, since there is no possibility of spontaneous closure. In cases not treated or with late diagnose, mortality is higher than $30 \%$ when associated with infection. We describe a case of anterior sacral meningocele, involving a 17-year-old girl, asymptomatic until then, with polymicrobial meningitis as initial clinical feature due to a rectothecal fistula.

\section{KEYWORDS}

Meningocele, fistula, meningitis.

1 Médicos-residentes do Serviço de Neurocirurgia do Hospital Celso Pierro da Pontifícia Universidade Católica de Campinas (PUC-Campinas), Campinas, SP, Brasil.

2 Médico-neurocirurgião chefe do Serviço de Neurocirurgia do Hospital Celso Pierro da PUC-Campinas, Campinas, SP, Brasil. 


\section{Introdução}

Meningoceles sacrais anteriores são raros exemplos de disrafismo espinhal. ${ }^{1-8}$ Originam-se da herniação do saco dural através de um defeito ósseo na parede anterior sacrococcígea. ${ }^{1-3,5,6,8-11}$ Essa condição foi descrita pela primeira vez em 1837 por Bryant apud Dios Seoane et al. ${ }^{1}$ Funayama et al. ${ }^{12} \mathrm{e}$ Hino et al. ${ }^{13} \mathrm{e}$ existem, aproximadamente, 250 casos relatados até os dias atuais. ${ }^{2,4,6,9,14,15}$

A tríade defeito ósseo, malformação anorretal e uma massa pré-sacral configura a síndrome de Currarino, ${ }^{1,6,8,10,14-20}$ e tal massa pode consistir de um tumor, de uma meningocele sacral anterior ou da associação de ambos. ${ }^{8,16}$ Apresenta incidência desconhecida ${ }^{15} \mathrm{e}$ acredita-se ser uma desordem de transmissão autossômica dominante. . $^{1,5,7,10,14,15,19-21}$

A maioria dos pacientes com tal síndrome apresenta constipação e sintomas vesicais compressivos. ${ }^{2,3,8-10,15,20,22}$ Raramente, ocorrem complicações devidas à formação de fístulas. ${ }^{915}$ Descrevemos um caso de meningocele sacral anterior acometendo uma jovem de 17 anos, assintomática até então, que apresentou meningite polimicrobiana como quadro inicial devido a uma fístula retotecal.

\section{Relato de caso}

Paciente de 17 anos, sexo feminino, admitida no pronto-socorro (PS) com história de cefaleia há um dia, occipital, com irradiação para todo o crânio, de forte intensidade, principalmente em ortostase, refratária ao tratamento com analgésicos simples, acompanhada de febre, sudorese e dificuldade progressiva de movimentação cervical e axial, além de sinais de meningismo no exame físico. Aventou-se a possibilidade de meningite, sendo realizada tomografia computadorizada (TC) de crânio, que demonstrou pneumoencéfalo. A análise liquórica evidenciou pleocitose neutrofílica, hipoglicorraquia e cocos Gram-positivos aos pares na bacterioscopia. A paciente e os familiares negavam traumas faciais ou cranianos anteriores.

Durante o início da internação, houve piora do nível de consciência, associada a sinais de sepse e culturas liquóricas positivas para E. coli, Pneumococo sp. e anaeróbios. Por causa do pneumoencéfalo sem explicação aparente e da observação de drenagem de secreção fluida hialina anal, foi solicitada ressonância nuclear magnética (RNM) de coluna lombossacral, que demonstrou meningocele sacral anterior volumosa com íntima relação com o reto (Figuras 1, 2 e 3). Após início da antibioticoterapia direcionada, a paciente apresentou melhora do quadro infeccioso e do nível de consciência, sendo encaminhada para tratamento cirúrgico.

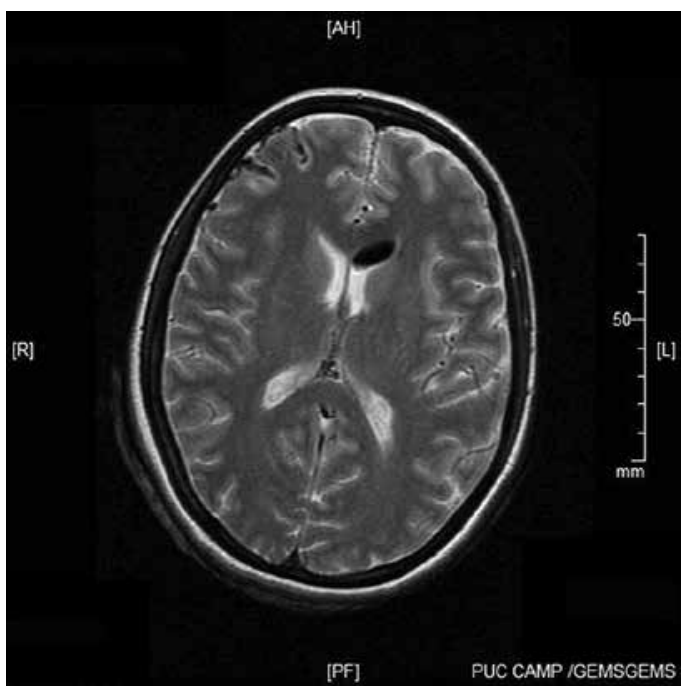

Figura 1 - Corte axial de RNM de crânio ponderada em T2 com presença de pneumoencéfalo.
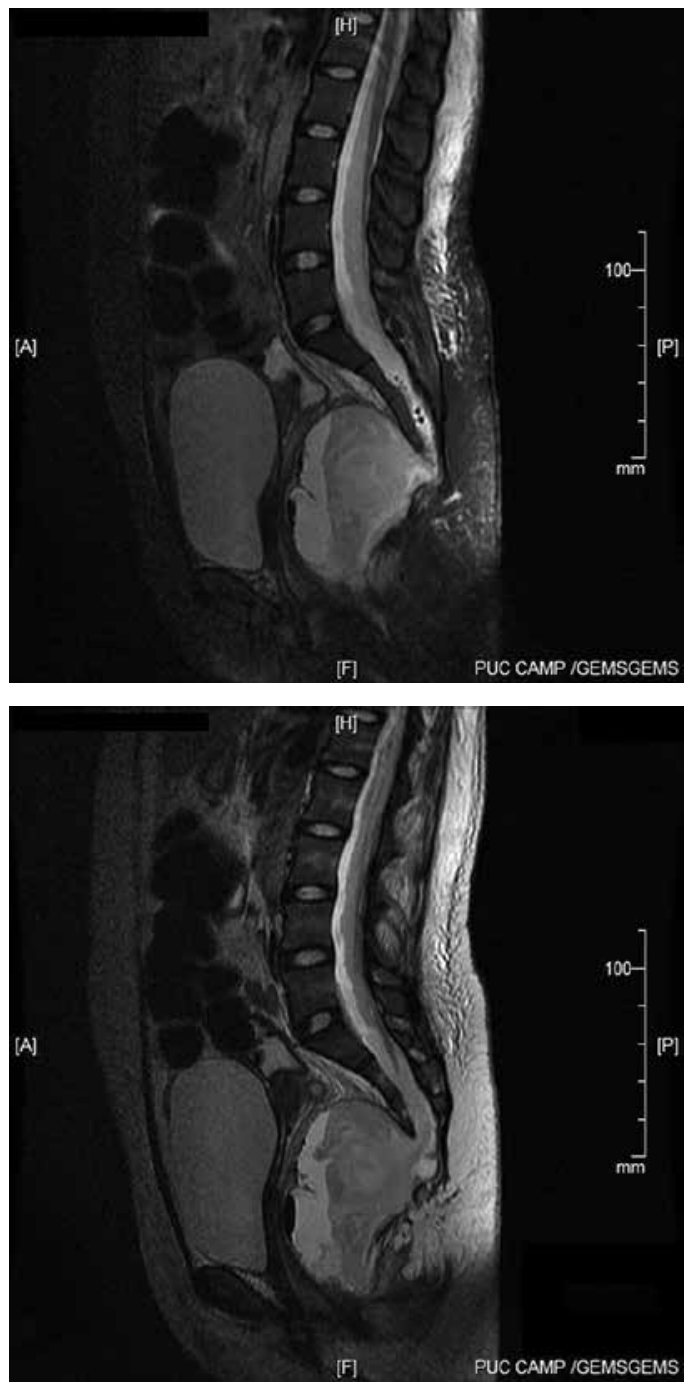

Figuras 2 e 3 - Cortes sagitais de RNM de pelve ponderadas em T2 demonstrando meningocele sacral anterior em intimo contato com o reto. 
O acesso neurocirúrgico foi realizado por via posterior com laminectomia sacral, durotomia, aspiração do conteúdo da meningocele (purulento e com presença de lamelas brancas nacaradas com pelos) e isolamento da meningocele e do saco tecal com sutura cuidadosa da dura-máter de ambos, seguido de colostomia realizada pela cirurgia geral. Houve melhora importante do quadro geral nos dias seguintes, no entanto se evidenciou fístula liquórica pelo aspecto da secreção presente no dreno. Optou-se por reabordagem para tratamento, que se mostrou efetiva. Três dias após o segundo procedimento, a paciente evoluiu com rebaixamento do nível de consciência e hidrocefalia comunicante, observada na TC de crânio, sendo submetida a derivação ventricular externa (DVE) de emergência e derivação ventriculoperitoneal (DVP) após negativação das culturas liquóricas. Recebeu alta hospitalar após 43 dias de internação com Glasgow Outcome Scale de 4.

Onze dias depois da alta, a paciente foi levada novamente ao PS pelos familiares com quadro súbito de rebaixamento do nível de consciência. Nova TC de crânio indicava recidiva da hidrocefalia, tendo sido realizada DVE de emergência e observada obstrução por grumos do cateter proximal da DVP. A paciente não apresentou melhora neurológica significativa após o novo procedimento, permanecendo não contactuante e com postura patológica. Após 57 dias, a paciente foi a óbito por causa de complicações infecciosas da internação prolongada em unidade de terapia intensiva e da DVE.

\section{Discussão}

A meningocele sacral anterior, além de ser uma condição rara em nosso meio, ocorrendo principalmente em pacientes do sexo feminino, ${ }^{1,2,4,9,12-14,17,19,20}$ possui várias formas de apresentação clínica, provavelmente relacionadas à compressão de estruturas circundantes, causando sintomas como constipação crônica, distúrbios urinários, dismenorreia, dores pélvicas ou lombares, além de hiperalgesia perineal e tônus reduzido no esfíncter anal e músculo detrusor. ${ }^{1,9}$ Os estigmas cutâneos clássicos e espinha bífida posterior podem estar ausentes. ${ }^{1}$

Em pacientes com defeitos anorretais associados, deve-se pensar na síndrome de Currarino, uma desordem autossômica dominante ligada à região $7 \mathrm{q} 36,1,14,15,19-21$ com vários relatos de transmissão familiar na literatura. ${ }^{1,16,20,21} \mathrm{~A}$ associação com medula ancorada e tumores como cistos dermoides, epidermoides, teratomas e lipomas também é descrita. ${ }^{1-3,8,14}$

O diagnóstico ainda é desafiador, apesar da evolução dos exames de imagem. TC e RNM são primordiais, sendo a última o melhor exame para caracterização. ${ }^{1,2,4,5,9,14,15} \mathrm{~A}$ radiografia simples pode ajudar demonstrando o defeito ósseo sacral e o sacro em "cimitarra", 2,5,14,17,20 sendo indicada para triagem de transmissão familiar. 12,14,20,21

A meningite é uma complicação rara ${ }^{1-3,10,15,22}$ e séria da meningocele sacral anterior. No caso relatado, a paciente apresentou infecção polimicrobiana e pneumoencéfalo como quadro clínico inicial da doença, decorrentes de uma fístula retotecal, condições relatadas em apenas dois artigos ${ }^{9,10}$ de língua inglesa. Outros artigos relatavam meningite recorrente. ${ }^{3,8,9,12}$ Além disso, a paciente apresentava, associado à malformação, um cisto dermoide, deixando o caso ainda mais incomum, uma vez que o tumor mais frequentemente associado é o teratoma. ${ }^{1,15,17}$

O tratamento deve ser cirúrgico, uma vez que não há possibilidade de fechamento espontâneo. ${ }^{1,2,8}$ Em casos não tratados ou com atraso diagnóstico, a mortalidade é superior a $30 \%$ quando associados a infecção. ${ }^{2,23}$ No entanto, durante a infância, a meningocele pode ser tratada de maneira conservadora em casos assintomáticos, por causa da possibilidade de dano iatrogênico causando incontinência e retenção urinária. ${ }^{15}$

Os princípios do tratamento incluem antibioticoterapia de amplo espectro na presença de infecção, esvaziamento ou ressecção da meningocele e fechamento do trajeto fistuloso, além de sutura, ressecção ou desvio do trânsito intestinal caso necessário.

A abordagem preconizada até a metade do século XX era a exposição e o fechamento transacral da meningocele com sutura primária do trajeto fistuloso, uma vez que a associação com procedimentos transabdominais resultava numa mortalidade superior a $27 \% .{ }^{1} \mathrm{Hoje}$, com a evolução de grampos e materiais cirúrgicos, a abordagem combinada pode ser utilizada com segurança. ${ }^{4,9}$ Sugere-se que seja usada a abordagem posterior para casos não complicados ${ }^{4,9,10,24} \mathrm{e}$ a anterior quando há complicações (infecções, aderências, grandes óstios, associação com massas pélvicas). ${ }^{10,13,24}$ Além disso, há técnicas endoscópicas relatadas com abordagem posterior e anterior, com ou sem procedimentos laparoscópicos. ${ }^{6,14,25}$

No caso apresentado, em associação com a cirurgia geral, optamos por laminectomia sacral, exclusão transdural do colo da meningocele anterior e colostomia temporária. Inicialmente, a ressecção da massa sacral seria realizada em um segundo tempo cirúrgico, após melhora do quadro infeccioso, juntamente com a reconstrução do trânsito intestinal. Entretanto, a RNM de controle demonstrou grande redução do volume da meningocele (Figura 4), que seria acompanhada ambulatorialmente para definição de necessidade de ressecção. 
Não há outros relatos, de acordo com a nossa pesquisa, de ocorrência de hidrocefalia aguda no pós-operatório precoce, mesmo nos casos associados com meningite. Consideramos tal processo infeccioso a provável causa da complicação, uma vez que a RNM de todo neuroeixo não demonstrava outras alterações congênitas que pudessem justificá-la (Figura 5).

O caso apresentado demonstra a importância de se considerar a meningocele sacral anterior em pacientes com meningite recorrente ou associada a pneumoencé-

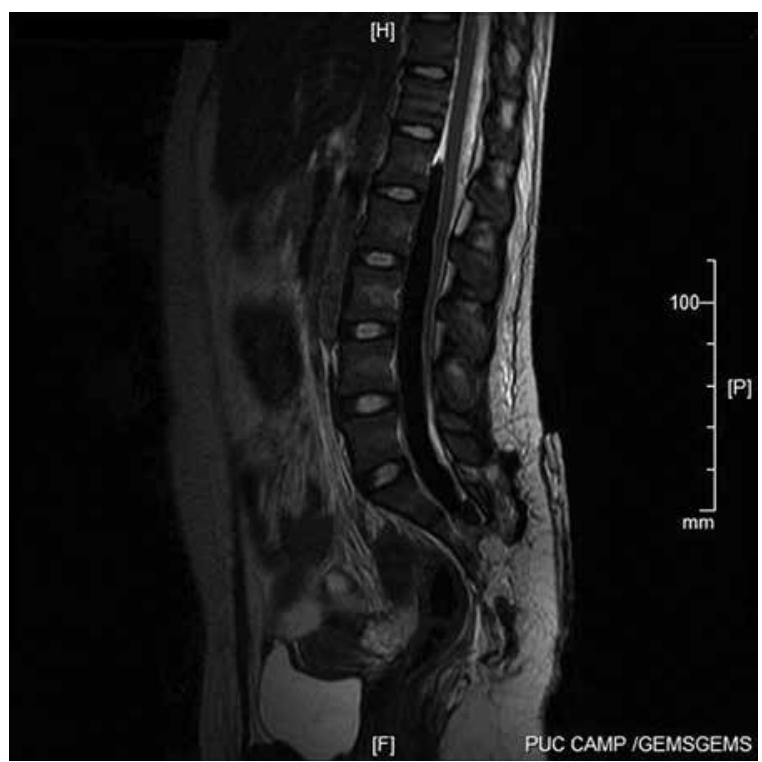

Figura 4 - Corte sagital de RNM de pelve ponderada em T2, realizada no pós-operatório, evidenciando importante redução da meningocele, reexpansão do reto e ar no saco dural.

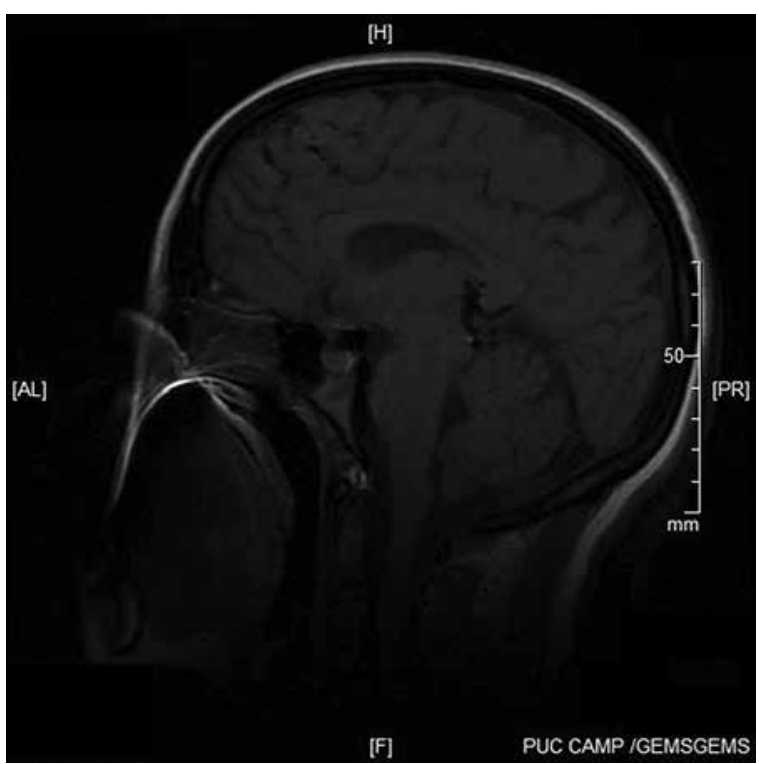

Figura 5 - Corte sagital de RNM de crânio ponderada em T1 sem evidências de alterações sugestivas de malformações. falo sem explicação evidente. A transmissão familiar, no caso da síndrome de Currarino, indica a necessidade de realização de radiografias para triagem nos parentes de primeiro grau. $\mathrm{O}$ tratamento deve ser cirúrgico, precoce quando associado à infecção e, apesar das diversas vias de abordagem existentes atualmente, não há consenso sobre qual é a melhor. A hidrocefalia é uma possível complicação, principalmente nos casos associados à infecção.

\section{Conflito de interesses}

Os autores declaram inexistência de conflito de interesses na realização deste trabalho.

\section{Referências}

1. Dios Seoane J, Amaro S, Fantini MG, Blanco A, López L, Rodiño A, et al. Anterior sacral meningocele with Currarino's syndrome: report of two cases. Neurocirugia (Astur). 2002;13(6):455-62.

2. Fitzpatrick MO, Taylor WA. Anterior sacral meningocele associated with a rectal fistula. Case report and review of the literature. J Neurosurg. 1999;91(Suppl 1):124-7.

3. Quigley MR, Schinco F, Brown JT. Anterior sacral meningocele with an unusual presentation. Case report. J Neurosurg. 1984;61(4):790-2.

4. Khan YA, Batool T, Rasool N, Jahan Y, Habib Q, Saddal NS. Anterior sacral meningocele. J Coll Physicians Surg Pak. 2010;20(5):337-8.

5. Mohta A, Das S, Jindal R. Anterior sacral meningocele presenting as constipation. J Pediatr Neurosci. 2011;6(1):40-3.

6. Jeon BC, Kim DH, Kwon KY. Anterior endoscopic treatment of a huge anterior sacral meningocele: technical case report. Neurosurgery. 2003;52(5):1231-3.

7. Marin-Sanabria EA, Nagashi T, Yamamoto $K$, Nakamura $Y$, Aihara $\mathrm{H}$, Kohmura E. Presacral meningocele associated with hereditary sacral agenesis and treated surgically: evaluation in three members of the same family. Neurosurgery. 2005;57(3):E597.

8. Antuña-Ramos A, García-Fructuoso G, Alamar-Abril M, Guillén-Quesada A, Costa-Clara JM. Occult anterior sacral meningocele. Neurocirugia (Astur). 2011;22(4):342-4.

9. Sánchez $A A$, Iglesias $C D$, López $C D$, Cecilia DM, Gómez JA, Barbadillo JG, et al. Rectothecal fistula secondary to an anterior sacral meningocele. J Neurosurg Spine. 2008;8(5):487-9.

10. Bergeron E, Roux A, Demers J, Vanier LE, Moore L. A 40-year-old woman with cauda equina syndrome caused by rectothecal fistula arising from an anterior sacral meningocele. Neurosurgery. 2010;67(5):E1464-7.

11. Lefere M, Verleyen N, Feys H, Somers JF. Anterior sacral meningocoele presenting as acute urinary retention. A case report. Acta Orthop Belg. 2009;75(6):855-7.

12. Funayama CA, Turcato MF, Moura-Ribeiro R, Rocha GM, Pina Neto JM, Moura-Ribeiro MV. Recurrent meningitis in a case of congenital anterior sacral meningocele 
and agenesis of sacral and coccygeal vertebrae. Arq Neuropsiquiatr. 1995;53(4):799-801.

13. Hino A, Taketomo S, Iwasaki T. Treatment of anterior sacral meningocele -case report. Neurol Med Chir (Tokyo). 1993;33(10):700-2.

14. Işik N, Balak N, Kircelli A, Göynümer G, Elmaci I. The shrinking of an anterior sacral meningocele in time following transdural ligation of its neck in a case of the Currarino triad. Turk Neurosurg. 2008;18(3):254-8.

15. Emans PJ, van Aalst J, van Heurn EL, Marcelis C, Kootstra G, Beets-Tan RG, et al. The Currarino triad: neurosurgical considerations. Neurosurgery. 2006;58(5):924-9.

16. Kansal R, Mahore A, Dange N, Kukreja S. Epidermoid cyst inside anterior sacral meningocele in an adult patient of Currarino syndrome manifesting with meningitis. Turk Neurosurg. 2012;22(5):659-61.

17. Haga $Y$, Cho H, Shinoda S, Masuzawa T. Recurrent meningitis associated with complete Currarino triad in an adult - case report. Neurol Med Chir (Tokyo). 2003;43(10):505-8.

18. Otagiri N, Matsumoto Y, Yoshida Y. Posterior sagittal approach for Currarino syndrome with anterior sacral meningocele: a case report. J Pediatr Surg. 2000;35(7):1112-4.

19. Samuel M, Hosie G, Holmes K. Currarino triad - diagnostic dilemma and a combined surgical approach. J Pediatr Surg. 2000;35(12):1790-4.
20. Gardner PA, Albright AL. "Like mother, like son:" hereditary anterior sacral meningocele. Case report and review of the literature. J Neurosurg. 2006;104(Suppl 2):138-42.

21. Kurosaki M, Kamitani H, Anno $\mathrm{Y}$, Watanabe $\mathrm{T}$, Hori T, Yamasaki T. Complete familial Currarino triad. Report of three cases in one family. J Neurosurg. 2001;94(Suppl 1):158-61.

22. Miletic D, Poljak I, Eskinja N, Valkovic P, Sestan B, TroseljVukic B. Giant anterior sacral meningocele presenting as bacterial meningitis in a previously healthy adult. Orthopedics. 2008;31(2):182.

23. Dyck P, Wilson CB. Anterior sacral meningocele. Case report. J Neurosurg. 1980;53(4):548-52.

24. Tani S, Okuda Y, Abe T. Surgical strategy for anterior sacral meningocele. Neurol Med Chir (Tokyo). 2003;43(4):204-9

25. Clatterbuck RE, Jackman SV, Kavoussi LR, Long DM. Laparoscopic treatment of an anterior sacral meningocele. Case illustration. J Neurosurg. 2000;92(Suppl 2):246.

\section{Endereço para correspondência}

Rodrigo de Almeida Simon Sola Av. Imperatriz Leopoldina, 550, torre 2, ap. 54, Bairro Vila Nova 13073-035 - Campinas, SP, Brasil Telefone: (19) 8166-5353

E-mail: rodssola@gmail.com 